\title{
LAW OF LARGE NUMBERS FOR CERTAIN CYLINDER FLOWS
}

\author{
PATRÍCIA CIRILO, YURI LIMA, AND ENRIQUE PUJALS
}

\begin{abstract}
We construct new examples of cylinder flows, given by skew product extensions of irrational rotations on the circle, that are ergodic and rationally ergodic along a subsequence of iterates. In particular, they exhibit law of large numbers. This is accomplished by explicitly calculating, for a subsequence of iterates, the number of visits to zero, and it is shown that such number has a gaussian distribution.
\end{abstract}

\section{INTRODUCTION}

The purpose of this paper is to construct examples of skew product extensions of irrational rotations of the additive circle $\mathbb{T}=\mathbb{R} / \mathbb{Z}$ exhibiting law of large numbers. More specifically, under some weak diophantine conditions on the irrational number $\alpha \in \mathbb{R}$, we construct roof functions $\phi: \mathbb{T} \rightarrow \mathbb{Z}$ for which the skew product

$$
\begin{aligned}
F: \mathbb{T} \times \mathbb{Z} & \longrightarrow \mathbb{T} \times \mathbb{Z} \\
(x, y) & \longmapsto(x+\alpha, y+\phi(x))
\end{aligned}
$$

is ergodic and rationally ergodic along a subsequence of iterates. This, in particular, implies that $F$ has a law of large numbers. See Subsection 2.4 for the proper definitions.

One must, first of all, observe that $F$ has a natural invariant measure, given by the product of the Lebesgue measure on $\mathbb{T}$ and the counting measure on $\mathbb{Z}$, and it is infinite. In this situation, classical theorems of ergodic theory are not valid. For instance, Birkhoff's averages converge to zero almost surely, and this leads us to the following question: what would be a good candidate for a Birkhoff-type theorem in this context? Denoting by $S_{n} \psi$ the Birkhoff sum of the $L^{1}$-function $\psi: \mathbb{T} \times \mathbb{Z} \rightarrow \mathbb{R}$, the most natural way is try to find a sublinear sequence $\left(a_{n}\right)$ of positive real numbers and consider the averages $S_{n} \psi / a_{n}$. However, by a result of J. Aaronson (see $\S 2.4$ of 3 ), there is never a universal sequence $\left(a_{n}\right)$ for which $S_{n} \psi / a_{n}$ converges pointwise to the right value. Nevertheless, Hopf's theorem (see $\S 2.2$ of [3]) is an indication that some sort of regularity might exist and it might still be possible, for a specific sequence $\left(a_{n}\right)$, that the averages oscillate without converging to zero or infinity and so one can hope for a summability method that smooths out the fluctuations and forces convergence. Such second order ergodic theorems were considered by J. Aaronson, M. Denker, and A. Fisher in [4.

Another attempt of obtaining a Birkhoff-type theorem has been made by Aaronson, in which he defined and constructed examples of rationally ergodic maps (see $\S 3.3$ of [3]). These maps possess a sort of Cèsaro-averaged version of convergence

Date: September 20, 2018.

2010 Mathematics Subject Classification. 37A05, 37A40.

Key words and phrases. cylinder flow, irrational rotation, law of large numbers, rationally ergodic, skew product, weakly homogeneous. 
in measure: there is a sequence $\left(a_{n}\right)$ such that, for every $L^{1}$-function $\psi$ and every subsequence $\left(n_{k}\right)$ of positive integers, there exists a further subsequence $\left(n_{l}\right)$ such that $S_{n_{l}} \psi(x) / a_{n_{l}}$ converges Cèsaro almost surely to $\int \psi$. This latter property is called weak homogeneity and the sequence $\left(a_{n}\right)$ is called a return sequence. Weak homogeneity implies the existence of law of large numbers (see Subsection 2.4).

A natural program of investigation regards two kinds of questions.

(i) What are the conservative, ergodic, rationally ergodic maps?

(ii) What fluctuations can the Birkhoff sums have?

Our goal in this work is to give contributions to these questions by constructing examples of the form (1.1) that are ergodic and rationally ergodic along a subsequence of iterates. Up to our knowledge, the first examples of ergodic cylinder flows were given by A. Krygin [16] and K. Schmidt 22. Their examples differ in nature. Krygin assures the existence, for any irrational $\alpha$, of a roof function $\phi$ for which $F$ is ergodic. Actually, there exist elegant categorical proofs that the set of pairs $(\alpha, \phi)$, in various different contexts, for which $F$ is ergodic forms a residual set. See [9] 15. On the other hand, Schmidt constructed an explicit example motivated by the theory of random walks. He considered $\alpha=(\sqrt{5}-1) / 4$ and the roof function equal to the Haar function defined in Section 3, which is actually the basis function for our example. Subsequent works 11, 10, 21] of J.-P. Conze and M. Keane and Schmidt himself extended the results to every irrational $\alpha$ and also to the larger class of roof functions

$$
\phi(x)=(\beta+1) \cdot \mathbb{1}_{\left[0, \frac{\beta}{\beta+1}\right)}(x)-\beta .
$$

There are many other works regarding this question. See for instance [6], [13, [14, [18, [19].

Regarding (ii), J. Aaronson and M. Keane further investigated Schmidt's example in 5. They studied the asymptotic behavior of the number of visits to zero and proved that the Birkhoff sums represent a sort of "deterministic random walk". In particular, they showed that if $\alpha$ is quadratic surd 1 then $F$ is rationally ergodic with return sequence $a_{n} \sim n / \sqrt{\log n}$.

Not much is known regarding rational ergodicity. There are actually few examples that have been proved to be rationally ergodic. See for instance [1, [2], [5], [7, [17, where this property is shown to hold in different contexts. With respect to cylinder flows given by skew products extensions of irrational rotations on the circle, the only known examples are those in [5].

The most significant contribution of our work is to construct a new class of cylinder flows that are rationally ergodic along a subsequence of iterates and, in particular, possess law of large numbers.

Theorem 1.1. For any $\alpha \in \mathbb{R}$ such that $\liminf _{q \rightarrow \infty} q\|q \alpha\|=0$, there exists a skew product

such that

$$
\begin{aligned}
F: \mathbb{T} \times \mathbb{Z} & \longrightarrow \mathbb{T} \times \mathbb{Z} \\
(x, y) & \longmapsto(x+\alpha, y+\phi(x))
\end{aligned}
$$

(a) $\phi$ belongs to $L^{p}(\mathbb{T})$, for every $p \geq 1$, and

(b) $F$ is ergodic.

\footnotetext{
${ }^{1}$ The irrational number $\alpha$ is quadratic surd if it satisfies a quadratic equation with integer coefficients.
} 
If $\alpha$ is also divisible, then

(c) F is rationally ergodic along a subsequence of iterates. In particular, it has a law of large numbers.

An irrational number $\alpha$ is divisible if it has a subsequence of continuants $\left(q_{n}\right)$ with a certain divisibility property and such that $\lim _{n \rightarrow \infty} q_{n}\left\|q_{n} \alpha\right\|=0$. See Subsection 2.2 for the specific definitions. It is worth noting that the set of $\alpha$ satisfying these two conditions has full Lebesgue measure, according to the content of Appendix B Thus, in contrast to [5], in which the set of parameters is countable, Theorem 1.1 holds for a set of parameters of full Lebesgue measure?

A remarkable feature of Theorem 1.1. is that the number of visits to zero along the iterates in which $F$ is rationally ergodic exhibits a gaussian distribution. The return sequence is given by $a_{q_{n+1}}=q_{n+1} / \sqrt{\pi n}$ and the normalized averages, described in equation (6.2), do not depend on the choice of $\alpha$ neither on the sequence $\left(q_{n}\right)$.

The roof function we construct is different in nature from the others used in this context. We consider the Haar function $T$ defined in Section 3 as a basis function and let

$$
\phi(x)=\frac{1}{2} \sum_{j \geq 1}\left[T\left(q_{j}(x+\alpha)\right)-T\left(q_{j} x\right)\right]
$$

for a specific chosen sequence of positive integers $\left(q_{n}\right)$. One can see $\phi$ as the limit of worser and worser coboundaries

$$
\phi_{n}(x)=\frac{1}{2} \sum_{j=1}^{n}\left[T\left(q_{j}(x+\alpha)\right)-T\left(q_{j} x\right)\right] .
$$

Observe that, if we just consider the coboundary $\phi_{n}$, the respective cylinder flow will not be ergodic and, moreover, will be conjugate to a rigid rotation. The increasing bad feature of each $\phi_{n}$ is what will guarantee that $\phi$ has the required properties. The sequence $\left(q_{n}\right)$ will be chosen via the continued fraction expansion of $\alpha$ and this is why the diophantine properties of $\alpha$ influence the dynamical properties of $F$. Even though $\phi$ is unbounded, the good feature of it is that we can explicitly calculate the number of visits to zero along a sequence of iterates of $F$. See Lemma 5.3 and Subsection 5.2 .

The paper is organized as follows. In Section 2 we introduce the basic notations and definitions as well as the necessary background for the sequel. Section 3 is devoted to the construction of the roof function $\phi$ and the related convergence issues. In Section 4 we establish the ergodicity of $F$ with the aide of the theory of random walks. To this matter, Appendix $\mathrm{A}$ treats the required results, adapted to our context. Section 5 calculates the number of returns of a generic point to its fiber, assuming that $\alpha$ is divisible. This in particular implies the second part of Theorem 1.1, which is the content of Section 6. In Appendix B, we enclose the results on continued fractions that allows us to state our results in the greatest possible generality.

Remark 1.2. In some sense, our construction resembles Anosov-Katok method of fast approximations developed in [8]. Indeed, the referred maps are obtained as limits of periodic maps and here we will also use this perspective (see Subsection

\footnotetext{
${ }^{2}$ In a previous version of this paper, Theorem 1.1 required stronger conditions on $\alpha$ for which the set of parameters has zero Lebesgue measure, but it was pointed to us that the proof works for any divisible irrational number.
} 
3.2). Another example that resembles ours is Hajian-Ito-Kakutani's map. See $\S 3.3$ of [20] for a detailed exposition of this map.

\section{Preliminaries}

2.1. General notation. Given a set $X, \# X$ denotes the cardinality of $X$. If $A$ is a subset of $X, \mathbb{1}_{A}: X \rightarrow\{0,1\}$ denotes the characteristic function of $A$ :

$$
\mathbb{1}_{A}(x)= \begin{cases}1, & \text { if } x \in A \\ 0, & \text { if } x \in X \backslash A .\end{cases}
$$

$\mathbb{Z}$ denotes the set of integers and $\mathbb{N}$ the set of positive integers. Each $n \in \mathbb{N}$ defines the ring $\mathbb{Z}_{n}$ of the residue classes module $n$. A complete residue system is a set $\left\{a_{1}, \ldots, a_{n}\right\}$ of integers such that $\left\{a_{1}, \ldots, a_{n}\right\}$ modulo $n$ is equal to $\mathbb{Z}_{n}$.

Given a real number $x,\lfloor x\rfloor$ and $\{x\}$ are the integer and fractional parts of $x$, respectively. Let $\|x\|$ be the distance from $x$ to the closest integer,

$$
\|x\|=\min \{\{x\}, 1-\{x\}\} .
$$

We use the following notation to compare the asymptotic of functions.

Definition 2.1. Let $f, g: \mathbb{N} \rightarrow \mathbb{R}$ be two real-valued functions. We say $f \lesssim g$ if there is a constant $C>0$ such that

$$
|f(n)| \leq C \cdot|g(n)|, \quad \forall n \in \mathbb{N} .
$$

If $f \lesssim g$ and $g \lesssim f$, we write $f \sim g$. We say $f \approx g$ if

$$
\lim _{n \rightarrow \infty} \frac{f(n)}{g(n)}=1 \text {. }
$$

Let $\mathbb{T}=\mathbb{R} / \mathbb{Z}$ denote the circle, parameterized by $[0,1)$, and let $d: \mathbb{T} \times \mathbb{T} \rightarrow \mathbb{R}$ be the induced distance function. For every $\alpha \in \mathbb{R}, R_{\alpha}: \mathbb{T} \rightarrow \mathbb{T}$ is the rotation $R_{\alpha} x=x+\alpha$.

Let $\lambda$ be the Lebesgue measure on $\mathbb{T}$ and $\mu$ the measure defined on the cylinder $\mathbb{T} \times \mathbb{Z}$ by $\mu=\lambda \times$ counting measure on $\mathbb{Z}$. Given a function $\psi: \mathbb{T} \rightarrow \mathbb{R}$, its $L^{p}$-norm with respect to $\lambda$ is defined as

$$
\|\psi\|_{p}=\left(\int_{\mathbb{T}}|\psi|^{p} d \lambda\right)^{1 / p}
$$

and the space of $L^{p}$-integrable functions as $L^{p}(\mathbb{T})$. Due to the index $p$, there will be no confusion between the integer norm $\|\cdot\|$ and the $L^{p}$-norm $\|\cdot\|_{p}$.

2.2. Continued fractions. Given an irrational number $\alpha$, consider its continued fraction expansion

$$
\alpha=a_{0}+\frac{1}{a_{1}+\frac{1}{a_{2}+\frac{1}{\ddots}}}:=\left[a_{0} ; a_{1}, a_{2}, \ldots\right],
$$

whose $n^{\text {th }}$-convergent is

$$
\alpha_{n}=\frac{p_{n}}{q_{n}}=\left[a_{0} ; a_{1}, a_{2}, \ldots, a_{n}\right], n \geq 0 .
$$


The $q_{n}$ are called the continuants. They give the best rational approximations to $\alpha$. More precisely, the approximation is equal to

$$
\left\|q_{n} \alpha\right\|=q_{n} \cdot\left|\alpha-\frac{p_{n}}{q_{n}}\right| .
$$

It is known, by Dirichlet's theorem, that

$$
\liminf _{q \rightarrow \infty} q\|q \alpha\| \leq 1
$$

for any $\alpha \in \mathbb{R}$. Let $\alpha$ be divisible if it has a sequence $\left(q_{n_{j}}\right)$ of continuants satisfying

$$
2 q_{n_{j}} \text { divides } q_{n_{j+1}} \text { and } \lim _{j \rightarrow \infty} q_{n_{j}}\left\|q_{n_{j}} \alpha\right\|=0 .
$$

The set of divisible numbers has full Lebesgue measure in $\mathbb{R}$. This is the content of Proposition B.1 which, in particular, guarantees that Theorem 1.1 is valid for Lebesgue almost every $\alpha \in \mathbb{R}$.

From now on, $\left(q_{n}\right)$ will denote a subsequence of (instead of all) continuants of $\alpha$ such that

$$
\lim _{n \rightarrow \infty} q_{n}\left\|q_{n} \alpha\right\|=0
$$

and, whenever $\alpha$ is divisible, this chosen sequence $\left(q_{n}\right)$ will also satisfy that $2 q_{n}$ divides $q_{n+1}$. We will also make constant use of the following conditions:

(CF1) For any $n \geq 1$,

$$
2 \sum_{j>n}\left\|q_{j} \alpha\right\|<\left\|q_{n} \alpha\right\|
$$

(CF2) For any $p \geq 1$,

$$
\sum_{j \geq 1} j^{p+1} \cdot\left\|q_{j} \alpha\right\|<\infty
$$

(CF3) For any $p \geq 1$,

$$
\sum_{j=1}^{n} j^{p+1} \cdot q_{j}<q_{n+1} \text { for } n>n(p) .
$$

(CF4) For any $n \geq 1$,

$$
\left(2^{n} \sum_{j=1}^{n-1} q_{j}\right) \cdot q_{n}\left\|q_{n} \alpha\right\|<1 .
$$

(CF5) For any $n \geq 1,\left\{\alpha, 2 \alpha, \ldots, q_{n+1} \alpha\right\}$ is $\left(\frac{1}{2 q_{n}}\right)^{2}$-dense in $\mathbb{T}$.

Condition (CF2) is always satisfied. Indeed,

$$
\sum_{j \geq 1} j^{p+1} \cdot\left\|q_{j} \alpha\right\|<\sum_{j \geq 1} \frac{j^{p+1}}{q_{j}}
$$

is bounded for every $p \geq 1$, because the exponential behavior of $q_{j}$ controls the polynomial behavior of $j^{p+1}$. (CF1), (CF3), (CF4) and (CF5) are assured by passing, if necessary, to a subsequence of $\left(q_{n}\right)$. 
2.3. Birkhoff sums. Let $\alpha \in \mathbb{R}, \phi: \mathbb{T} \rightarrow \mathbb{R}$ a $L^{1}$-measurable function and $F$ defined as in (1.1). The dynamics of $F$ is intimately connected to the cocycle $S(\alpha, \phi): \mathbb{T} \times \mathbb{Z} \rightarrow \mathbb{R}$ defined as the Birkhoff sums of $\phi$ with respect to the rotation $R_{\alpha}$ :

$$
S(\alpha, \phi)(x, n)= \begin{cases}\sum_{k=0}^{n-1} \phi(x+k \alpha) & \text {,if } n \geq 1 \\ 0 & \text {,if } n=0 \\ -\sum_{k=1}^{-n} \phi(x-k \alpha) & \text {,if } n<0 .\end{cases}
$$

For simplicity, we denote $S(\alpha, \phi)(\cdot, n): \mathbb{T} \rightarrow \mathbb{R}$ by $S_{n}(\alpha, \phi)$. From now on, we assume $\int_{\mathbb{T}} \phi d \lambda=0$. This in particular implies conservativity of the associated cylinder flow. See $\S 8.1$ of $[3]$.

2.4. Law of large numbers. As observed in the introduction, there is no Birkhofftype theorem for ergodic and infinite measure-preserving systems. Nevertheless, one can hope for a law of large numbers.

Definition 2.2. A law of large numbers for a conservative ergodic measure-preserving system $(X, \mathcal{A}, \mu, F)$ is a function $L:\{0,1\}^{\mathbb{N}} \rightarrow[0, \infty]$ such that, for any $A \in \mathcal{A}$, the equality

$$
L\left(\mathbb{1}_{A}(x), \mathbb{1}_{A}(F x), \mathbb{1}_{A}\left(F^{2} x\right), \ldots\right)=\mu(A)
$$

holds for $\mu$-almost every $x \in X$.

One can see the function $L$ as a sort of blackbox: given the input of hittings of a generic point $x \in X$ to a fixed set $A \in \mathcal{A}$, the output is the measure of $A$. There are systems with no law of large number 3 . On the other hand, there are some conditions that guarantee its existence.

Given $A \in \mathcal{A}$, let $S_{n}(A): X \rightarrow \mathbb{N}$ be the Birkhoff sum of the characteristic function $\mathbb{1}_{A}$ with respect to $F$.

Definition 2.3. A conservative ergodic measure-preserving system $(X, \mathcal{A}, \mu, F)$ is called rationally ergodic along a subsequence of iterates if there is a set $A \in \mathcal{A}$ with $0<\mu(A)<\infty$ satisfying the Renyi inequality

$$
\int_{A} S_{n_{k}}(A)^{2} d \mu \lesssim\left(\int_{A} S_{n_{k}}(A) d \mu\right)^{2}
$$

for some increasing sequence $\left(n_{k}\right)$ of positive integers.

We note the above definition differs from the original one [1, since the Renyi inequality is asked to hold, instead of all positive integers, only for a subsequence of them.

Definition 2.4. A conservative ergodic measure-preserving system $(X, \mathcal{A}, \mu, F)$ is called weakly homogeneous if there is a sequence $\left(a_{n_{k}}\right)$ of positive real numbers such

\footnotetext{
${ }^{3}$ Squashable transformations, for example, have no law of large numbers. See $\S 8.4$ of $[3]$.
} 
that, for all $\phi \in L^{1}(X, \mathcal{A}, \mu)$,

$$
\frac{1}{N} \sum_{k=1}^{N} \frac{1}{a_{n_{k}}} \sum_{j=0}^{n_{k}-1} \phi\left(F^{j} x\right) \longrightarrow \int_{X} \phi d \mu
$$

for $\mu$-almost every $x \in X$.

$\left(a_{n_{k}}\right)$ is called a return sequence of $F$ and it is unique up to asymptotic equality. Aaronson proved that rational ergodicity along a subsequence of iterates implies weak homogeneity with

$$
a_{n_{k}}=\frac{1}{\mu(A)^{2}} \int_{A} S_{n_{k}}(A) d \mu=\frac{1}{\mu(A)^{2}} \sum_{j=0}^{n_{k}-1} \mu\left(A \cap F^{-j} A\right) .
$$

See $\S 3.3$ of 3 . Observe that weak homogeneity defines a law of large numbers $L:\{0,1\}^{\mathbb{N}} \rightarrow[0, \infty]$ by

$$
L\left(x_{0}, x_{1}, \ldots\right)=\left\{\begin{aligned}
\lim _{N \rightarrow \infty} \frac{1}{N} \sum_{k=1}^{N} \frac{1}{a_{n_{k}}} \sum_{j=0}^{n_{k}-1} x_{j}, & \text { if the limit exists }, \\
0, & \text { otherwise. }
\end{aligned}\right.
$$

The goal of this work is to construct examples of cylinder flows given by skew product extensions of irrational rotations on the circle that are ergodic and rationally ergodic along a subsequence of iterates and, therefore, have law of large numbers.

\section{Construction of Roof FunCtion $\phi$}

Let $T: \mathbb{T} \rightarrow \mathbb{Z}$ be the Haar function, defined as

$$
T(x)=\left\{\begin{aligned}
1, & \text { if } x \in\left[0, \frac{1}{2}\right) \\
-1, & \text { if } x \in\left[\frac{1}{2}, 1\right) .
\end{aligned}\right.
$$

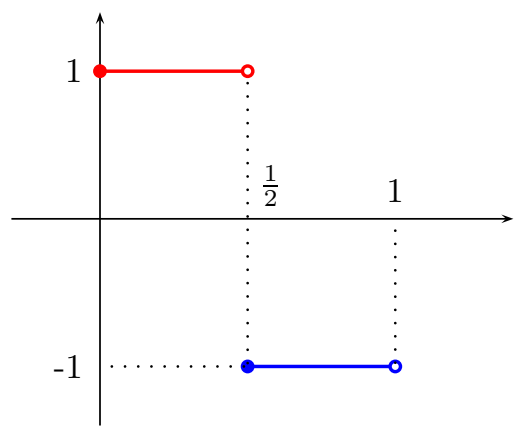

Figure 1: the graph of $T$.

Let $\alpha \in \mathbb{R}$ and $\left(q_{n}\right)$ its associated subsequence of continuants, that is, satisfying (2.1) and (CF1) to (CF4). For each $j \geq 1$, let $T_{j}: \mathbb{T} \rightarrow \mathbb{Z}$ be the dilation of $T$ by 
$q_{j}$, that is, $T_{j}(x)=T\left(q_{j} x\right)$, where $q_{j} x$ (and any expression appearing as argument of $T$ ) is taken modulo 1 . The function we will consider is

$$
\phi(x)=\frac{1}{2} \sum_{j \geq 1}\left[T_{j}(x+\alpha)-T_{j}(x)\right] .
$$

First of all, it is not clear that this defines a $L^{1}$-measurable function. The proof of this fact depends on a couple of auxiliary lemmas.

Lemma 3.1. Let $q$ be a positive integer and $\beta, \gamma \in \mathbb{T}$. Then the set

$$
\{x \in \mathbb{T} ; T(q x+\beta) \neq T(q x+\gamma)\}
$$

has Lebesgue measure equal to $2\|\beta-\gamma\|$.

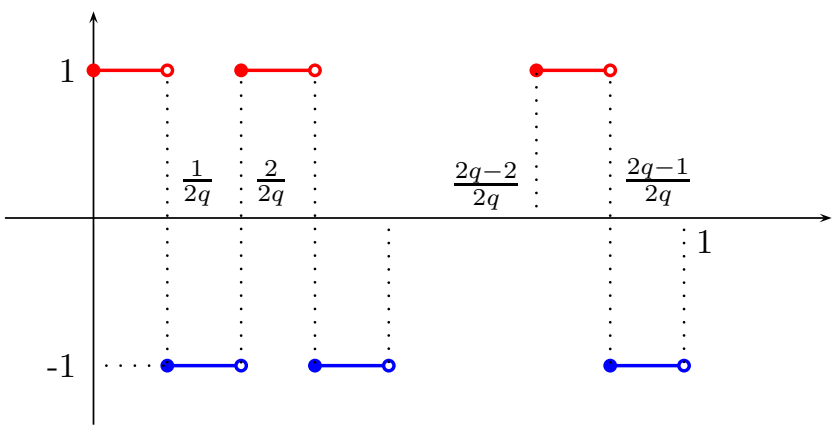

Figure 2: the graph of $x \mapsto T(q x)$.

Proof. Just observe that, because the Lebesgue measure is preserved under the map $x \mapsto q x,\{x \in \mathbb{T} ; T(q x+\beta) \neq T(q x+\gamma)\}$ has Lebesgue measure equal to the Lebesgue measure of the set $\{x \in \mathbb{T} ; T(x+\beta) \neq T(x+\gamma)\}$, which is equal to $2\|\beta-\gamma\|$.

Lemma 3.2. Let $\left(q_{n}\right)$ be a sequence of positive integers and $\left(\beta_{n}\right),\left(\gamma_{n}\right)$ sequences in $\mathbb{T}$. If $\psi: \mathbb{T} \rightarrow \mathbb{Z}$ is defined by

$$
\psi(x)=\frac{1}{2} \sum_{j \geq 1}\left[T\left(q_{j} x+\beta_{j}\right)-T\left(q_{j} x+\gamma_{j}\right)\right],
$$

then

$$
\|\psi\|_{p}^{p} \leq 2 \sum_{j \geq 1} j^{p+1} \cdot\left\|\beta_{j}-\gamma_{j}\right\|
$$

Proof. Assume the right hand side of (3.1) is finite. In particular, $\sum\left\|\beta_{j}-\gamma_{j}\right\|$ is convergent. For each $n \geq 1$, let

$$
\Lambda_{n}=\left\{x \in \mathbb{T} ; T\left(q_{j} x+\beta_{j}\right)=T\left(q_{j} x+\gamma_{j}\right), \forall j>n\right\} .
$$

In $\Lambda_{n}$, we have

$$
\psi(x)=\frac{1}{2} \sum_{j=1}^{n}\left[T\left(q_{j} x+\beta_{j}\right)-T\left(q_{j} x+\delta_{j}\right)\right] .
$$

The complement of $\Lambda_{n}$ is defined by the property that $T\left(q_{j} x+\beta_{j}\right) \neq T\left(q_{j} x+\gamma_{j}\right)$ for some $j>n$. By Lemma 3.1, its Lebesgue measure is at most $2 \sum_{j>n}\left\|\beta_{j}-\gamma_{j}\right\|$. Then the sequence of functions $\left(\psi_{n}\right)$ given by $\psi_{n}=\psi \cdot \mathbb{1}_{\Lambda_{n}}$ converges pointwise to 
$\psi$. By Fatou's Lemma, the result will follow if we manage to prove (3.1) for each $\psi_{n}$.

Fixed $n \geq 1$, we have

$$
\left|\psi_{n}(x)\right| \leq \sum_{j=1}^{n}\left|\frac{T\left(q_{j} x+\beta_{j}\right)-T\left(q_{j} x+\gamma_{j}\right)}{2}\right|, \forall x \in \mathbb{T} .
$$

Define, for each $m \in\{1, \ldots, n\}$, the set

$$
A_{m}=\left\{x \in \mathbb{T} ; \sum_{j=1}^{n}\left|\frac{T\left(q_{j} x+\beta_{j}\right)-T\left(q_{j} x+\gamma_{j}\right)}{2}\right|=m\right\} .
$$

If we further define, for each $j \in\{1, \ldots, n\}$, the set

$$
A_{m}^{j}=\left\{x \in A_{m} ; j \text { is the largest index such that } T\left(q_{j} x+\beta_{j}\right) \neq T\left(q_{j} x+\gamma_{j}\right)\right\},
$$

then

$$
A_{m}=\bigsqcup_{j=m}^{n} A_{m}^{j}
$$

Each $A_{m}^{j}$ is contained in the set $\left\{x \in \mathbb{T} ; T\left(q_{j} x+\beta_{j}\right) \neq T\left(q_{j} x+\gamma_{j}\right)\right\}$ and so, by Lemma 3.1 , its Lebesgue measure is at most $2\left\|\beta_{j}-\gamma_{j}\right\|$. Summing up this estimate in $j$ and $m$, we obtain that

$$
\begin{aligned}
\left\|\psi_{n}\right\|_{p}^{p} & =\int_{\mathbb{T}}\left|\psi_{n}\right|^{p} d \lambda \\
& \leq \sum_{m=1}^{n} m^{p} \cdot \lambda\left(A_{m}\right) \\
& \leq 2 \sum_{m=1}^{n} m^{p} \sum_{j=m}^{n}\left\|\beta_{j}-\gamma_{j}\right\| \\
& \leq 2 \sum_{m=1}^{n} \sum_{j=m}^{n} j^{p} \cdot\left\|\beta_{j}-\gamma_{j}\right\| \\
& \leq 2 \sum_{j \geq 1} j^{p+1} \cdot\left\|\beta_{j}-\gamma_{j}\right\|,
\end{aligned}
$$

thus establishing (3.1) for $\psi_{n}$.

Lemma 3.2 will be used repeatedly in the next subsections, the first time being to prove that $\phi_{n}$, as defined in (1.3), converges to $\phi$.

3.1. $\left(\phi_{n}\right)$ converges to $\phi$ in $L^{p}(\mathbb{T})$. By Lemma 3.2 ,

$$
\begin{aligned}
\left\|\phi-\phi_{n}\right\|_{p}^{p} & =\left\|\frac{1}{2} \sum_{j>n}\left[T\left(q_{j} x+q_{j} \alpha\right)-T\left(q_{j} x\right)\right]\right\|_{p}^{p} \\
& \leq 2 \sum_{j>n} j^{p+1} \cdot\left\|q_{j} \alpha\right\|
\end{aligned}
$$

which, by condition (CF2), goes to zero as $n$ goes to infinity. 
3.2. $\left(\tilde{\phi}_{n}\right)$ converges to $\phi$ in $L^{p}(\mathbb{T})$. In order to make the calculations of Section 5. in which estimates on the return map of $F$ will be given, we need to approximate $\phi$ by something easier to manage with. We will approximate $\phi$ not by $\phi_{n}$, but by its "rational" truncated versions $\tilde{\phi}_{n}$, defined as

$$
\tilde{\phi}_{n}(x)=\frac{1}{2} \sum_{j=1}^{n}\left[T_{j}\left(x+\alpha_{n+1}\right)-T_{j}(x)\right] .
$$

Let us prove that the functions $\tilde{\phi}_{n}$ converge to $\phi$ in $L^{p}(\mathbb{T})$ for any $p \geq 1$. This follows by another application of Lemma 3.2. Indeed, as

$$
\phi_{n}(x)-\tilde{\phi}_{n}(x)=\frac{1}{2} \sum_{j=1}^{n}\left[T\left(q_{j} x+q_{j} \alpha\right)-T\left(q_{j} x+q_{j} \alpha_{n+1}\right)\right],
$$

we have

$$
\begin{aligned}
\left\|\tilde{\phi}_{n}-\phi_{n}\right\|_{p}^{p} & \leq 2 \sum_{j=1}^{n} j^{p+1} \cdot\left\|q_{j} \alpha-q_{j} \alpha_{n+1}\right\| \\
& \leq 2 \sum_{j=1}^{n} j^{p+1} \cdot q_{j} \cdot\left|\alpha-\alpha_{n+1}\right| \\
& =\frac{2\left\|q_{n+1} \alpha\right\|}{q_{n+1}} \sum_{j=1}^{n} j^{p+1} \cdot q_{j} \\
& \leq 2\left\|q_{n+1} \alpha\right\|
\end{aligned}
$$

where in the last inequality we used (CF3).

\section{ERGODICITY}

4.1. Branches and plateaux. We call a branch of $T_{j}$ any of the branches $\left[\frac{i}{q_{j}}, \frac{i+1}{q_{j}}\right)$, $i=0,1, \ldots, q_{j}-1$, of the expanding map $x \mapsto q_{j} x$. Each branch of $T_{j}$ decomposes itself in two subintervals $\left[\frac{2 i}{2 q_{j}}, \frac{2 i+1}{2 q_{j}}\right)$ and $\left[\frac{2 i+1}{2 q_{j}}, \frac{2 i+2}{2 q_{j}}\right)$, each of them called a plateau of $T_{j}$, in which $T_{j}$ is constant (see figure 2 ). The first will be called a positive plateau and the second a negative plateau.

Let $I_{j}(x)$ denote the plateau of $T_{j}$ containing $x$ and

$$
m_{n}(x):=T_{1}(x)+\cdots+T_{n}(x), \quad n \geq 1 .
$$

If $\left(q_{n}\right)$ satisfies the divisibility condition, then clearly $I_{1}(x) \supset I_{2}(x) \supset \cdots$ and so we have the implication

$$
y \in I_{n}(x) \Longrightarrow m_{n}(x)=m_{n}(y) \text {. }
$$

This is also true if, instead of the divisibility condition, $\left(q_{n}\right)$ satisfies Lemma A.1 More specifically, using the notation of Appendix A

$$
I_{n_{0}}(x) \supset I_{n_{0}+1}(x) \supset \cdots \quad \text { whenever } x \in \Omega_{n_{0}}^{\infty} .
$$

For such a fixed $x$, there is a positive integer $n_{1}=n_{1}(x)$ such that

$$
\begin{aligned}
I_{n}(x) & \subset I_{1}(x), \ldots, I_{n_{0}}(x) \\
\Longrightarrow & \bigcap_{j=1}^{n} I_{j}(x)=I_{n}(x)
\end{aligned}
$$


for every $n \geq n_{1}$ and so (4.1) remains valid. We will use this condition below.

4.2. Ergodicity. We will prove ergodicity in two steps.

Step 1. For any $A \subset \mathbb{T} \times\{0\}$ of positive measure, the union $\bigcup_{n \geq 1} F^{n} A$ contains $\mathbb{T} \times\{0\}$ modulo zero.

Step 2. $F(\mathbb{T} \times\{0\}) \cap(\mathbb{T} \times\{1\})$ and $F(\mathbb{T} \times\{0\}) \cap(\mathbb{T} \times\{-1\})$ have positive measure.

Once this is done, it is clear that $F$ will be ergodic. Actually, let $A \subset \mathbb{T} \times \mathbb{Z}$ be $F$-invariant with positive measure. We can assume that $A$ has positive measure when restricted to the fiber $\mathbb{T} \times\{0\}$. By Step $1, A$ has full measure in $\mathbb{T} \times\{0\}$. By Step 2, $A$ has also positive measure in both fibers $\mathbb{T} \times\{1\}$ and $\mathbb{T} \times\{-1\}$. Applying repeatedly Steps 1 and 2 , we conclude that $A$ has full measure in $\mathbb{T} \times \mathbb{Z}$. Step 1 will follow from the next

Lemma 4.1. Let $A_{1}, A_{2} \subset \mathbb{T} \times\{0\}$ have positive $\mu$-measure. Then there is $n \geq 1$ such that the intersection $F^{n} A_{1} \cap A_{2}$ has positive $\mu$-measure.

To prove Lemma 4.1. we will localize $A_{1}$ and $A_{2}$ to subsets in which $\phi$ and $\phi_{n}$ coincide, and actually their Birkhoff sums up to the order $q_{n+1}$. Letting $\mathcal{D}=$ $\{0,1 / 2\}$, this set is defined as

$$
\Lambda_{n}=\left\{x \in \mathbb{T} ; d\left(q_{j} x, \mathcal{D}\right)>q_{j}\left\|q_{j} \alpha\right\| \text { for } j>n\right\} .
$$

Note that

$$
d\left(q_{j}(x+k \alpha), q_{j} x\right)=\left\|k q_{j} \alpha\right\|=k\left\|q_{j} \alpha\right\| \leq q_{j}\left\|q_{j} \alpha\right\|
$$

whenever $j>n$ and $k=1, \ldots, q_{n+1}$. This implies that

$$
F^{k}(x, 0)=\left(x+k \alpha, S_{k}\left(\alpha, \phi_{n}\right)(x)\right), x \in \Lambda_{n}, k=1, \ldots, q_{n+1} .
$$

Observe that the $\Lambda_{n}$ 's form an ascending chain of subsets of $\mathbb{T}$ and that $\mathbb{T} \backslash \Lambda_{n}$ has Lebesgue measure at most $\sum_{j>n} q_{j}\left\|q_{j} \alpha\right\|$. We can suppose, after passing to a subsequence 4 , that this sum is smaller than $2^{-n}$.

Proof of Lemma 4.1. Define the set

$$
\Sigma_{n}=\left\{x \in \mathbb{T} ; d\left(x, \partial I_{j}(x)\right)>\left(\frac{1}{2 q_{j}}\right)^{2} \text { for } j>n\right\} .
$$

The sequence $\left(\Sigma_{n}\right)$ also forms an ascending chain of subsets of $\mathbb{T}$ and 5

$$
\lambda\left(\mathbb{T} \backslash \Sigma_{n}\right) \leq \sum_{j>n} \frac{1}{q_{j}} .
$$

This together with the fact that $\lambda\left(\Lambda_{n}\right), \lambda\left(\Omega_{n}^{\infty}\right) \rightarrow 1$ as $n \rightarrow \infty$ allows us to take $n_{0} \geq 1$ large enough and assume that

(i) $A_{1} \subset \Lambda_{n_{0}}$,

(ii) $A_{1}, A_{2} \subset \Sigma_{n_{0}}$ and

(iii) $A_{1}, A_{2} \subset \Omega_{n_{0}}^{\infty}$.

\footnotetext{
${ }^{4}$ Here is where Theorem 1.1 requires that $\liminf _{q \rightarrow \infty} q\|q \alpha\|=0$.

${ }^{5}$ For each plateau of $T_{j}$, we remove two intervals of length $\left(\frac{1}{2 q_{j}}\right)^{2}$. As $T_{j}$ has $2 q_{j}$ plateaux, the estimate is correct.
} 
By the Lebesgue differentiation theorem, let $x_{1}, x_{2}$ be points of density for $A_{1}, A_{2}$, respectively. Now choose $n_{1} \geq 1$ large enough (see Subsection 4.1) such that

(iv) $\bigcap_{j=1}^{n} I_{j}\left(x_{i}\right)=I_{n}\left(x_{i}\right)$ for every $n \geq n_{1}$ and $i=1,2$.

Finally, let $n \geq n_{0}, n_{1}$ such that

(v) $m_{n}\left(x_{1}\right)=m_{n}\left(x_{2}\right)$ and

(vi) $\lambda\left(A_{i} \cap\left(x_{i}-\left(\frac{1}{2 q_{n}}\right)^{2}, x_{i}+\left(\frac{1}{2 q_{n}}\right)^{2}\right)\right)>\frac{3}{4} \cdot 2\left(\frac{1}{2 q_{n}}\right)^{2}$ for $i=1,2$.

The existence of such $n$ is assured by Lemma A.1 and the fact that $x_{i}$ is a point of density for $A_{i}$. For simplicity, let

$$
\tilde{A}_{i}=A_{i} \cap\left(x_{i}-\left(\frac{1}{2 q_{n}}\right)^{2}, x_{i}+\left(\frac{1}{2 q_{n}}\right)^{2}\right), \quad i=1,2 .
$$

By (ii), $\tilde{A}_{i} \subset I_{n}\left(x_{i}\right)$. Now use (CF5) to choose $k \in\left\{1,2, \ldots, q_{n+1}\right\}$ such that

$$
d\left(x_{1}+k \alpha, x_{2}\right)<\left(\frac{1}{2 q_{n}}\right)^{2}
$$

The proof of the lemma will follow from the next two claims.

Claim 1. The set $\left(\tilde{A}_{1}+k \alpha\right) \cap \tilde{A}_{2} \subset \mathbb{T}$ has positive Lebesgue measure.

Indeed, (4.2) implies that the union $\left(\tilde{A}_{1}+k \alpha\right) \cup \tilde{A}_{2}$ is contained in an interval of length $3 \cdot\left(\frac{1}{2 q_{n}}\right)^{2}$ and so, by (vi),

$$
\begin{aligned}
\lambda\left(\left(\tilde{A}_{1}+k \alpha\right) \cap \tilde{A}_{2}\right) & =\lambda\left(\tilde{A}_{1}+k \alpha\right)+\lambda\left(\tilde{A}_{2}\right)-\lambda\left(\left(\tilde{A}_{1}+k \alpha\right) \cup \tilde{A}_{2}\right) \\
& >\frac{3}{2} \cdot\left(\frac{1}{2 q_{n}}\right)^{2}+\frac{3}{2} \cdot\left(\frac{1}{2 q_{n}}\right)^{2}-3 \cdot\left(\frac{1}{2 q_{n}}\right)^{2} \\
& =0 .
\end{aligned}
$$

Claim 2. The set $F^{k}\left(\tilde{A}_{1} \times\{0\}\right) \cap\left(\tilde{A}_{2} \times\{0\}\right) \subset \mathbb{T} \times \mathbb{Z}$ has positive $\mu$-measure.

It is enough to prove that $S_{k}(\alpha, \phi)(x)=0$ for every $x$ satisfying Claim 1 . By (i), $x \in \Lambda_{n_{0}} \subset \Lambda_{n}$ and so

$$
S_{k}(\alpha, \phi)(x)=S_{k}\left(\alpha, \phi_{n}\right)(x)=m_{n}(x+k \alpha)-m_{n}(x) .
$$

Observe that

- $x \in \tilde{A}_{1} \subset I_{n}\left(x_{1}\right)$ and so (iv) guarantees that $m_{n}(x)=m_{n}\left(x_{1}\right)$.

- $x+k \alpha \in \tilde{A}_{2} \subset I_{n}\left(x_{2}\right)$. Using (iv) again, $m_{n}(x+k \alpha)=m_{n}\left(x_{2}\right)$.

By assumption (v) it follows that $S_{k}(\alpha, \phi)(x)=0$ for every $x$ satisfying Claim 1. This concludes the proof of Claim 2 and also from the lemma.

We thus obtained Step 1. Step 2 follows from Lemma 3.1. Indeed, for $s \in$ $\{-1,1\}$, the set of points $x \in \mathbb{T}$ such that

- $T_{1}(x+\alpha)=T_{1}(x)+2 s$ and

- $T_{j}(x+\alpha)=T_{j}(x)$ for $j>1$

has Lebesgue measure at least $\left\|q_{1} \alpha\right\|-2 \sum_{j>1}\left\|q_{j} \alpha\right\|$, which is positive by (CF1). This concludes the proof of ergodicity. 
Remark 4.2. The argument of this section indeed shows that $F$ is regular in the sense of [21].

\section{Counting the number of Returns}

Throughout this and the next section, we assume $\alpha$ is divisible and its subsequence $\left(q_{n}\right)$ of continuants satisfies

$$
2 q_{n} \text { divides } q_{n+1} \text { and } \lim _{n \rightarrow \infty} q_{n}\left\|q_{n} \alpha\right\|=0 .
$$

Let $A=\mathbb{T} \times\{0\}$. The purpose of this section is to count the number of returns of an arbitrary point $(x, 0) \in A$ to $A$ via the map $F$. More specifically, identifying $A$ with $\mathbb{T}$, we want to investigate the function $S_{q_{n+1}}^{F}: \mathbb{T} \rightarrow \mathbb{N}$ defined as

$$
S_{q_{n+1}}^{F}(x)=\sum_{k=1}^{q_{n+1}}\left(\mathbb{1}_{A} \circ F^{k}\right)(x, 0) .
$$

In the next section we will apply the estimates obtained here to establish Theorem 1.1 .

As remarked before, we will not directly calculate $S_{q_{n+1}}^{F}$. Instead, we consider the rational truncated versions of $F$ defined by the skew product

$$
\begin{aligned}
\tilde{F}_{n}: \mathbb{T} \times \mathbb{Z} & \longrightarrow \mathbb{T} \times \mathbb{Z} \\
(x, y) & \longmapsto\left(x+\alpha_{n+1}, y+\tilde{\phi}_{n}(x)\right),
\end{aligned}
$$

where $\tilde{\phi}_{n}$ is given by (3.2), and calculate the value of $S_{q_{n+1}}^{\tilde{F}_{n}}: \mathbb{T} \rightarrow \mathbb{N}$ given by

$$
S_{q_{n+1}}^{\tilde{F}_{n}}(x)=\sum_{k=1}^{q_{n+1}}\left(\mathbb{1}_{A} \circ \tilde{F}_{n}^{k}\right)(x, 0) .
$$

By approximation, $S_{q_{n+1}}^{F}$ and $S_{q_{n+1}}^{\tilde{F}_{n}}$ coincide for a large subset of $\mathbb{T}$ and then we will have the value of the former function in this large set.

This section is organized as follows. In Subsection 5.1, we calculate the distribution of $S_{q_{n+1}}^{\tilde{F}_{n}}$. After that, Subsection 5.2 establishes the distribution of $S_{q_{n+1}}^{F}$.

5.1. The function $S_{q_{n+1}}^{\tilde{F}_{n}}$. Observe that

$$
\tilde{F}_{n}^{k}(x, 0)=\left(x+k \alpha_{n+1}, S_{k}\left(\alpha_{n+1}, \tilde{\phi}_{n}\right)(x)\right)
$$

so that $\tilde{F}_{n}^{k}(x, 0)$ belongs to $A$ if and only if

$$
S_{k}\left(\alpha_{n+1}, \tilde{\phi}_{n}\right)(x)=0 \Longleftrightarrow m_{n}\left(x+k \alpha_{n+1}\right)=m_{n}(x) .
$$

Then

$$
S_{q_{n+1}}^{\tilde{F}_{n}}(x)=\#\left\{1 \leq k \leq q_{n+1} ; m_{n}\left(x+k \alpha_{n+1}\right)=m_{n}(x)\right\} .
$$

The idea to calculate the above cardinality is: for each sequence $\mathbf{s}=\left(s_{1}, \ldots, s_{n}\right) \in$ $\{-1,1\}^{n}$, consider the set

$$
B_{\mathbf{s}}=\left\{1 \leq k \leq q_{n+1} ; T_{j}\left(x+k \alpha_{n+1}\right)=s_{j} \text { for } j=1, \ldots, n\right\} .
$$


If we manage to prove that each $B_{\mathbf{s}}$ has the same cardinality (independent of $\mathbf{s}$ ), it must be equal to $q_{n+1} / 2^{n}$. Then

$$
\begin{aligned}
S_{q_{n+1}}^{\tilde{F}_{n}}(x) & =\sum_{\substack{\mathbf{s} \in\{-1,1\} n \\
s_{1}+\cdots+s_{n}=m_{n}(x)}} \# B_{\mathbf{s}} \\
& =\frac{q_{n+1}}{2^{n}} \cdot \#\left\{\mathbf{s} \in\{-1,1\}^{n} ; s_{1}+\cdots+s_{n}=m_{n}(x)\right\}
\end{aligned}
$$

and so

$$
S_{q_{n+1}}^{\tilde{F}_{n}}(x)=\frac{q_{n+1}}{2^{n}}\left(\begin{array}{c}
n \\
\frac{n+m_{n}(x)}{2}
\end{array}\right) .
$$

This is indeed the case. Roughly speaking, we prove that each $B_{\mathbf{s}}$ has the same cardinality by interpreting $m_{n}(x)$ as a random walk. More specifically, we consider the intermediate sets

$$
B_{\left(s_{1}, \ldots, s_{i}\right)}=\left\{1 \leq k \leq q_{n+1} ; T_{j}\left(x+k \alpha_{n+1}\right)=s_{j} \text { for } j=1, \ldots, i\right\}
$$

and associate to them a binary tree as follows:

- The root of the tree is $B=\left\{1,2, \ldots, q_{n+1}\right\}$.

- $B_{\left(s_{1}, \ldots, s_{i}\right)}$ has exactly two descendants: $B_{\left(s_{1}, \ldots, s_{i}, 1\right)}$ and $B_{\left(s_{1}, \ldots, s_{i},-1\right)}$.

Observe that

$$
B_{\left(s_{1}, \ldots, s_{i}\right)}=B_{\left(s_{1}, \ldots, s_{i}, 1\right)} \sqcup B_{\left(s_{1}, \ldots, s_{i},-1\right)}
$$

so that, at each level $i$, the union of the $B_{\left(s_{1}, \ldots, s_{i}\right)}$ 's is equal to $B$. We will prove that, in each subdivision of $B_{\left(s_{1}, \ldots, s_{i}\right)}$, half of the elements belong to $B_{\left(s_{1}, \ldots, s_{i}, 1\right)}$ and the other half to $B_{\left(s_{1}, \ldots, s_{i},-1\right)}$. Once this is done, (5.1) will be established.

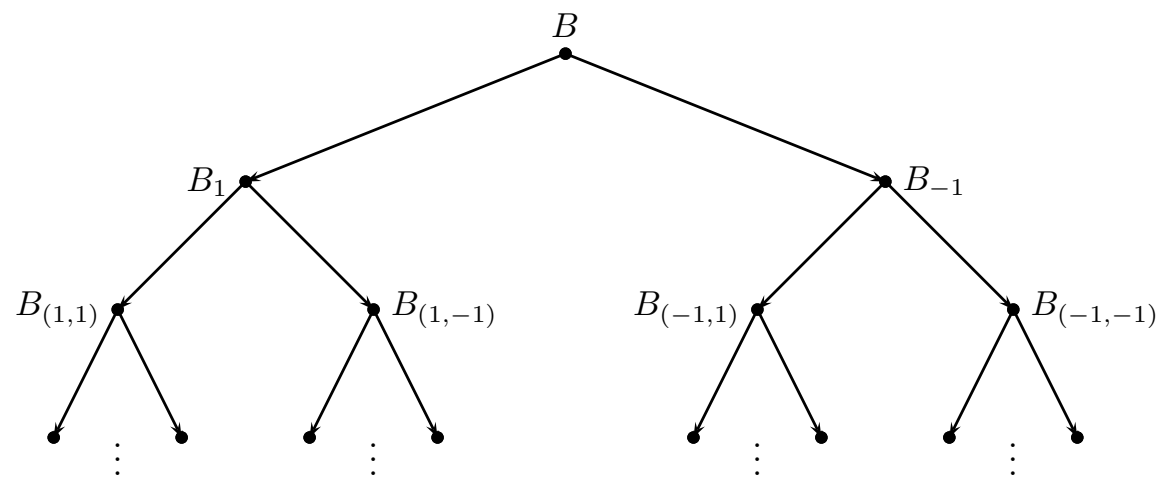

Figure 3: the binary tree.

Fix $x \in \mathbb{T}$. The idea is to see $k$ as a variable $z \in \mathbb{R}$ and to prove that the evaluations of the functions $z \mapsto T_{j}\left(\alpha_{n+1} z+x\right), j=1,2, \ldots, n$, along the integers $1,2, \ldots, q_{n+1}$ satisfy the required binary property. Each of these functions is periodic, with period equal to

$$
\pi_{j}=\frac{1}{q_{j} \alpha_{n+1}}=\frac{q_{n+1} / q_{j}}{p_{n+1}}=: \frac{u_{j}}{v} .
$$


Better than this, consider the functions given by the composition with the dilation $z \mapsto z / v$, defined as

$$
\begin{aligned}
\psi_{j}: \mathbb{R} & \longrightarrow \mathbb{R} \\
z & \longmapsto T\left(\frac{z}{u_{j}}+q_{j} x\right), j=1,2, \ldots, n,
\end{aligned}
$$

whose period is equal to $u_{j} \in \mathbb{Z}$. We thus want to investigate $\psi_{1}, \ldots, \psi_{n}$ along the integers $v, 2 v, \ldots, u_{1} v$. Observe that

- $\left\{v, 2 v, \ldots, u_{1} v\right\}$ is a complete residue system modulo $u_{1}$,

- $u_{n}$ is even and $u_{j}$ is a multiple of $2 u_{j+1}$ for $j=1, \ldots, n-1$, and

- for a set $x \in \mathbb{T}$ of full Lebesgue measure, $\psi_{1}, \ldots, \psi_{n}$ are continuous in $\mathbb{Z}$ (i.e. none of their discontinuities is an integer).

These are the assumptions we make below.

Proposition 5.1. Let $\psi_{j}: \mathbb{R} \rightarrow \mathbb{R}$ be a periodic function with period $u_{j} \in \mathbb{Z}$, $j=1, \ldots, n$. Assume that

(a) $u_{n}$ is even and $u_{j}$ is a multiple of $2 u_{j+1}$ for $j=1, \ldots, n-1$, and

(b) there are $z_{1}, \ldots, z_{n} \in \mathbb{R} \backslash \mathbb{Q}$ such that

$$
\left.\psi_{j}\right|_{\left[z_{j}, z_{j}+\frac{u_{j}}{2}\right)} \equiv 1 \text { and }\left.\psi_{j}\right|_{\left[z_{j}+\frac{u_{j}}{2}, z_{j}+u_{j}\right)} \equiv-1
$$

$$
\text { for } j=1, \ldots, n \text {. }
$$

Let $R$ be a complete residue system modulo $u_{1}$. Then, for any sequence $\left(s_{1}, \ldots, s_{n}\right) \in$ $\{-1,1\}^{n}$,

$$
\#\left\{k \in R ; \psi_{j}(k)=s_{j} \text { for } j=1, \ldots, n\right\}=\frac{u_{1}}{2^{n}} .
$$

The proof is by induction on $n$. Let us give an idea of why this must be true. Assume that $x=0$ and that, instead of being interested in the behavior of $\psi_{1}, \ldots, \psi_{n}$ along integers, we want to compute the Lebesgue measure of the set

$$
\left\{z \in\left[0, u_{1}\right) ; \psi_{j}(k)=s_{j} \text { for } j=1, \ldots, n\right\} .
$$

For $n=1$, we have

$$
\begin{aligned}
\left\{z \in\left[0, u_{1}\right) ; \psi_{1}(k)=1\right\} & =\left[0, \frac{u_{1}}{2}\right) \\
\left\{z \in\left[0, u_{1}\right) ; \psi_{1}(k)=-1\right\} & =\left[\frac{u_{1}}{2}, u_{1}\right) .
\end{aligned}
$$

For $n=2$, observe that in both intervals $\left[0, \frac{u_{1}}{2}\right),\left[\frac{u_{1}}{2}, u_{1}\right)$ the function $\psi_{2}$ alternately changes sign at each interval of length $u_{2} / 2$ so that, for any $s_{1}, s_{2} \in\{-1,1\}$, $\left\{z \in\left[0, u_{1}\right) ; \psi_{j}(k)=s_{j}\right.$ for $\left.j=1,2\right\}$ is the union of $u_{1} / 2 u_{2}$ intervals of length $u_{2} / 2$. For arbitrary $n,(5.3)$ is the union of $u_{1} / 2^{n-1} u_{n}$ intervals of length $u_{n} / 2$ each and so its Lebesgue measure is equal to $u_{1} / 2^{n}$. Proposition 5.1 is nothing but a discrete version of this. In order to prove it, we just have to make sure that none of the discontinuities of $\psi_{1}, \ldots, \psi_{n}$ are integer. This is accomplished by condition (b).

The next auxiliary lemma constitutes the basis of induction.

Lemma 5.2. Let $\psi: \mathbb{R} \rightarrow \mathbb{R}$ be a function with period $u \in \mathbb{Z}$ such that

(a) $u$ is even and

(b) there is $z \in \mathbb{R} \backslash \mathbb{Q}$ such that

$$
\left.\psi\right|_{\left[z, z+\frac{u}{2}\right)} \equiv 1 \text { and }\left.\psi\right|_{\left[z+\frac{u}{2}, z+u\right)} \equiv-1 .
$$


Let $R$ be a complete residue system modulo $u$. Then

$$
\#\{k \in R ; \psi(k)=1\}=\#\{k \in R ; \psi(k)=-1\}=\frac{u}{2} .
$$

Proof. Consider the sets

$$
\begin{aligned}
& \Psi_{+}=\left\{i \in \mathbb{Z} ; i \in\left[z, z+\frac{u}{2}\right)\right\} \quad(\bmod u) \text { and } \\
& \Psi_{-}=\left\{i \in \mathbb{Z} ; i \in\left[z+\frac{u}{2}, z+u\right)\right\} \quad(\bmod u) .
\end{aligned}
$$

It is clear that $\Psi_{+} \cup \Psi_{-}=\mathbb{Z}_{u}$ and that $\# \Psi_{+}=\# \Psi_{-}=u / 2$. Also, $\psi(k)=1$ if and only if $k \equiv i(\bmod u)$ for some $i \in \Psi_{+}$. Because $R$ is a complete residue system module $u$, the lemma is proved.

Proof of Proposition 5.1. The basis of induction is Lemma 5.2. It remains to prove the inductive step. We will do the case $n=2$, as the general inductive step follows the same lines of ideas, except that more notation would have to be introduced.

Let $\psi_{1}, \psi_{2}: \mathbb{R} \rightarrow \mathbb{R}$ be two functions satisfying the conditions of the proposition. For $j=1,2$, consider the equipartition of $\mathbb{Z}_{u_{j}}$ by the subsets

$$
\begin{array}{ll}
\Psi_{+}^{j}=\left\{i \in \mathbb{Z} ; i \in\left[z_{j}, z_{j}+\frac{u_{j}}{2}\right)\right\} & \left(\bmod u_{j}\right) \quad \text { and } \\
\Psi_{-}^{j}=\left\{i \in \mathbb{Z} ; i \in\left[z_{j}+\frac{u_{j}}{2}, z_{j}+u_{j}\right)\right\} & \left(\bmod u_{j}\right) .
\end{array}
$$

For $s_{1}, s_{2} \in\{-1,1\} \cong\{-,+\}$,

$$
\left\{\begin{array} { l } 
{ \psi _ { 1 } ( k ) = s _ { 1 } } \\
{ \psi _ { 2 } ( k ) = s _ { 2 } }
\end{array} \Longleftrightarrow \left\{\begin{array}{lll}
k \equiv i_{1} & \left(\bmod u_{1}\right) & \text { for } i_{1} \in \Psi_{s_{1}}^{1} \\
k \equiv i_{2} & \left(\bmod u_{2}\right) & \text { for } i_{2} \in \Psi_{s_{2}}^{2} .
\end{array}\right.\right.
$$

Because $u_{2}$ divides $u_{1}$, residue classes module $u_{1}$ define residue classes module $u_{2}$. This implies that the above congruences are equivalent to

$$
\left\{\begin{array}{cc}
k \equiv i_{1} & \left(\bmod u_{1}\right) \text { for } i_{1} \in \Psi_{s_{1}}^{1} \\
i_{1} \equiv i_{2} & \left(\bmod u_{2}\right) \text { for } i_{2} \in \Psi_{s_{2}}^{2}
\end{array}\right.
$$

and then we want to count the cardinality of the set

$$
\left\{\begin{array}{lll}
k \in R ; & k \equiv i_{1} \quad\left(\bmod u_{1}\right) & \text { for } i_{1} \in \Psi_{s_{1}}^{1} \\
i_{1} \equiv i_{2} & \left(\bmod u_{2}\right) & \text { for } i_{2} \in \Psi_{s_{2}}^{2}
\end{array}\right\}
$$

Each residue class modulo $u_{2}$ is equal to the union of $u_{1} / u_{2}$ residue classes modulo $u_{1}$. More specifically,

$$
i_{1} \equiv i_{2} \quad\left(\bmod u_{2}\right) \Longleftrightarrow i_{1} \equiv i_{2}, i_{2}+u_{2}, \ldots, i_{2}+\left(u_{1}-u_{2}\right) \quad\left(\bmod u_{1}\right)
$$

so that (5.4) is equal to the union

$$
\bigcup_{i_{2} \in \Psi_{s_{2}}^{2}}\left\{i_{2}, i_{2}+u_{2}, \ldots, i_{2}+\left(u_{1}-u_{2}\right)\right\} \cap \Psi_{s_{1}}^{1} .
$$


Independent of $i_{2}$, half of the residue classes $i_{2}, i_{2}+u_{2}, \ldots, i_{2}+\left(u_{1}-u_{2}\right)$ modulo $u_{1}$ belong to $\Psi_{+}^{1}$ and half to $\Psi_{-}^{1}$. Thus

$$
\begin{aligned}
\#\left\{k \in R ; \psi_{1}(k)=s_{1} \text { and } \psi_{2}(k)=s_{2}\right\} & =\# \Psi_{s_{2}}^{2} \cdot \frac{u_{1}}{2 u_{2}} \\
& =\frac{u_{2}}{2} \cdot \frac{u_{1}}{2 u_{2}} \\
& =\frac{u_{1}}{4},
\end{aligned}
$$

where in the second equality we used Lemma 5.2 .

In our context, Proposition 5.1 is translated to

Lemma 5.3. For every $m \in\{-n, \ldots, n\}$ with the same parity of $n$,

$$
S_{q_{n+1}}^{\tilde{F}_{n}}(x)=\frac{q_{n+1}}{2^{n}}\left(\begin{array}{c}
n \\
\frac{n+m}{2}
\end{array}\right)
$$

for a set of $x \in \mathbb{T}$ of Lebesgue measure $\left(\begin{array}{c}n \\ \frac{n+m}{2}\end{array}\right) / 2^{n}$.

Proof. Let $u_{j}=q_{n+1} / q_{j}$ for $j=1, \ldots, n$ and apply Proposition 5.1 to the functions in (5.2). The random variable $x \in \mathbb{T} \mapsto m_{n}(x)$ has the same distribution as the $n$-th step of a simple random walk in $\mathbb{Z}$, and so the equality $m_{n}(x)=m$ holds in a set of Lebesgue measure $\left(\frac{n}{n+m} 2\right) / 2^{n}$, for every $m \in\{-n, \ldots, n\}$ with the same parity of $n$.

5.2. The function $S_{q_{n+1}}^{F}$. It is a matter of fact that $\phi$ and $\tilde{\phi}_{n}$ coincide in a large set, and actually their Birkhoff sums up to the order $q_{n+1}$. This set is defined by those points simultaneously satisfying

(i) $T_{j}(x+k \alpha)=T_{j}\left(x+k \alpha_{n+1}\right)$ for $j=1, \ldots, n$ and $k=1, \ldots, q_{n+1}$, and

(ii) $d\left(q_{j} x, \mathcal{D}\right)>q_{j}\left\|q_{j} \alpha\right\|$ for $j>n$.

Call this set $\Lambda_{n}$. Note that

$$
d\left(q_{j}(x+k \alpha), q_{j} x\right)=\left\|k q_{j} \alpha\right\|=k\left\|q_{j} \alpha\right\| \leq q_{j}\left\|q_{j} \alpha\right\|
$$

whenever $j>n$ and $k=1, \ldots, q_{n+1}$ and so (ii) implies $T_{j}(x+k \alpha)=T_{j}(x)$. This equality guarantees that

$$
\begin{aligned}
& F^{k}(x, 0)=\left(x+k \alpha, S_{k}\left(\alpha, \tilde{\phi}_{n}\right)(x)\right) \quad \text { for } x \in \Lambda_{n}, k=1, \ldots, q_{n+1} \\
& \Longrightarrow \quad S_{q_{n+1}}^{F}(x)=S_{q_{n+1}}^{\tilde{F}_{n}}(x) \quad \text { for } x \in \Lambda_{n} .
\end{aligned}
$$

By Lemma 3.1, the Lebesgue measure of points not satisfying (i) is at most

$$
\sum_{\substack{1 \leq k \leq q_{n+1} \\ 1 \leq j \leq n}}\left\|k q_{j}\left(\alpha-\alpha_{n+1}\right)\right\|<\left|\alpha-\alpha_{n+1}\right| \cdot q_{n+1}^{2} \cdot \sum_{j=1}^{n} q_{j}<2^{-n-1},
$$

where in the last inequality we used (CF4). The points not satisfying (ii) have Lebesgue measure at most $\sum_{j>n} q_{j}\left\|q_{j} \alpha\right\|<2^{-n}$ and so

$$
\lambda\left(\mathbb{T} \backslash \Lambda_{n}\right)<2^{-n+1} .
$$

The above estimate will be used in the next section.

\footnotetext{
${ }^{6}$ Remember we are assuming $\sum_{j>n} q_{j}\left\|q_{j} \alpha\right\|<2^{-n}$.
} 


\section{RAtional ERGODicity ALONG $\left(q_{n}\right)$}

It remains to prove that $F$ satisfies the Renyi inequality along $\left(q_{n}\right)$. This will be obtained via the estimates of Section 5 More specifically, we first prove, as a consequence of Lemma 5.3 , that the rational truncated version $\tilde{F}_{n}$ of $F$ satisfies the Renyi inequality in the time $q_{n+1}$, uniformly in $n$. We then prove that $\left\|S_{q_{n+1}}^{F}\right\|_{1} \approx$ $\left\|S_{q_{n+1}}^{\tilde{F}_{n}}\right\|_{1}$ and $\left\|S_{q_{n+1}}^{F}\right\|_{2} \approx\left\|S_{q_{n+1}}^{\tilde{F}_{n}}\right\|_{2}$, which allows us to push the Renyi inequality to $F$.

6.1. Renyi inequality for $\tilde{F}_{n}$. By Lemma 5.3 ,

$$
\begin{aligned}
\left\|S_{q_{n+1}}^{\tilde{F}_{n}}\right\|_{1} & =\int_{\mathbb{T}} S_{q_{n+1}}^{\tilde{F}_{n}} d \lambda \\
& =\sum_{\substack{-n \leq m \leq n \\
m \equiv n(\bmod 2)}}\left[\frac{q_{n+1}}{2^{n}}\left(\begin{array}{c}
n \\
\frac{n+m}{2}
\end{array}\right)\right] \cdot\left[\frac{1}{2^{n}}\left(\begin{array}{c}
n \\
\frac{n+m}{2}
\end{array}\right)\right] \\
& =\frac{q_{n+1}}{2^{2 n}} \sum_{i=0}^{n}\left(\begin{array}{c}
n \\
i
\end{array}\right) \\
& =\frac{q_{n+1}}{2^{2 n}}\left(\begin{array}{c}
2 n \\
n
\end{array}\right) \\
& \approx \frac{q_{n+1}}{2^{2 n}} \cdot \frac{2^{2 n}}{\sqrt{\pi n}} \\
& =\frac{q_{n+1}}{\sqrt{\pi n}},
\end{aligned}
$$

where in the fifth passage we used Stirling's formuld to estimate the central binomial coefficient. On the other hand,

$$
\begin{aligned}
\left\|S_{q_{n+1}}^{\tilde{F}_{n}}\right\|_{2}^{2} & =\sum_{\substack{-n \leq m \leq n \\
m \equiv n(\bmod 2)}}\left[\frac{q_{n+1}}{2^{n}}\left(\begin{array}{c}
n \\
\frac{n+m}{2}
\end{array}\right)\right]^{2} \cdot\left[\frac{1}{2^{n}}\left(\begin{array}{c}
n \\
\frac{n+m}{2}
\end{array}\right)\right] \\
& =\frac{q_{n+1}^{2}}{2^{3 n}} \sum_{i=0}^{n}\left(\begin{array}{c}
n \\
i
\end{array}\right)^{3} \\
& \leq \frac{q_{n+1}^{2}}{2^{3 n}}\left(\begin{array}{c}
n \\
\frac{n}{2}
\end{array}\right) \sum_{i=0}^{n}\left(\begin{array}{c}
n \\
i
\end{array}\right)^{2} \\
& =\frac{q_{n+1}^{2}}{2^{3 n}}\left(\begin{array}{c}
n \\
\frac{n}{2}
\end{array}\right)\left(\begin{array}{c}
2 n \\
n
\end{array}\right) \\
& \approx \sqrt{2} \cdot \frac{q_{n+1}^{2}}{\pi n}
\end{aligned}
$$

and therefore

$$
\frac{\left\|S_{q_{n+1}}^{\tilde{F}_{n}}\right\|_{2}}{\left\|S_{q_{n+1}}^{\tilde{F}_{n}}\right\|_{1}} \lesssim \frac{\sqrt[4]{2} \cdot \frac{q_{n+1}}{\sqrt{\pi n}}}{\frac{q_{n+1}}{\sqrt{\pi n}}} \lesssim 1 .
$$

\footnotetext{
${ }^{7}$ Stirling's formula states that $n ! \approx \sqrt{2 \pi n}\left(\frac{n}{e}\right)^{n}$.
} 
6.2. Renyi inequality for $F$. Using (5.6),

$$
\left|\left\|S_{q_{n+1}}^{F}\right\|_{1}-\left\|S_{q_{n+1}}^{\tilde{F}_{n}}\right\|_{1}\right| \leq \int_{\mathbb{T} \backslash \Lambda_{n}}\left|S_{q_{n+1}}^{F}-S_{q_{n+1}}^{\tilde{F}_{n}}\right| d \lambda<q_{n+1} \cdot 2^{-n+1}
$$

and so

$$
\left|\frac{\left\|S_{q_{n+1}}^{F}\right\|_{1}}{\left\|S_{q_{n+1}}^{\tilde{F}_{n}}\right\|_{1}}-1\right| \lesssim \frac{q_{n+1} \cdot 2^{-n+1}}{\frac{q_{n+1}}{\sqrt{\pi n}}} \approx 0,
$$

proving that $\left\|S_{q_{n+1}}^{F}\right\|_{1} \approx\left\|S_{q_{n+1}}^{\tilde{F}_{n}}\right\|_{1}$. Analogously,

$$
\left|\frac{\left\|S_{q_{n+1}}^{F}\right\|_{2}^{2}}{\left\|S_{q_{n+1}}^{\tilde{F}_{n}}\right\|_{2}^{2}}-1\right| \lesssim \frac{q_{n+1}^{2} \cdot 2^{-n+1}}{\frac{q_{n+1}^{2}}{\pi n}} \approx 0
$$

and so $\left\|S_{q_{n+1}}^{F}\right\|_{2} \approx\left\|S_{q_{n+1}}^{\tilde{F}_{n}}\right\|_{2}$. These two estimates, together with (6.1), guarantee that

$$
\left\|S_{q_{n+1}}^{F}\right\|_{2} \lesssim\left\|S_{q_{n+1}}^{F}\right\|_{1},
$$

thus establishing the Renyi inequality for $F$ along $\left(q_{n}\right)$. This concludes the proof of Theorem 1.1 .

We calculate the return sequence $\left(a_{q_{n}}\right)$ for $F$. According to (2.3), it is given by

$$
a_{q_{n+1}}=\left\|S_{q_{n+1}}^{F}\right\|_{1} \approx\left\|S_{q_{n+1}}^{\tilde{F}_{n}}\right\|_{1} \approx \frac{q_{n+1}}{\sqrt{\pi n}}
$$

and so for a fixed $x \in \Lambda_{n}$ the normalized averages

$$
\frac{S_{q_{n+1}}^{F}(x)}{a_{q_{n+1}}} \approx \frac{\frac{q_{n+1}}{2^{n}}\left(\begin{array}{c}
n \\
\frac{n+m_{n}(x)}{2}
\end{array}\right)}{\frac{q_{n+1}}{\sqrt{\pi n}}}=\frac{\left(\begin{array}{c}
n \\
\frac{n+m_{n}(x)}{2}
\end{array}\right)}{\frac{2^{n}}{\sqrt{\pi n}}} \approx \sqrt{2} \cdot \frac{\left(\begin{array}{c}
n \\
\frac{n+m_{n}(x)}{2}
\end{array}\right)}{\left(\begin{array}{c}
n \\
\frac{n}{2}
\end{array}\right)}
$$

do not depend on the choice of $\alpha$ neither on the sequence $\left(q_{n}\right)$.

\section{Final Comments}

1. In order to obtain ergodic cylinder flows on $\mathbb{T} \times \mathbb{R}$, one can consider a similar construction to ours with roof function as in (1.2), where $\beta \in \mathbb{R}$ is irrational. In this case, the image of the map is contained in $\mathbb{T} \times\{m+n \beta ; m, n \in \mathbb{Z}\}$, which is dense in $\mathbb{T} \times \mathbb{R}$.

2. So far, all the examples of rationally ergodic cylinder flows use non-continuous roof functions. Another natural program is to construct examples with continuous (even $C^{1}$ and $C^{\infty}$ ) roof functions. It seems to us that the same approach developed in the present paper might work if one can interpret the sequence $\left(m_{n}\right)$ as defined in Subsection 4.1 from a random perspective. 


\section{ACKNOWLEDGMENTS}

The authors are thankful to IMPA for the excellent ambient during the preparation of this manuscript and to Alejandro Kocsard, François Ledrappier, Carlos Gustavo Moreira and Omri Sarig for valuable comments and suggestions. This research was possible due to the support of CNPq-Brazil, Faperj-Brazil and J. Palis 2010 Balzan Prize for Mathematics.

\section{APPEndix A. RANDOM WALKS}

Let $T: \mathbb{T} \rightarrow \mathbb{Z}$ as defined in Section 3, For each sequence of positive integers $\left(q_{n}\right)$, we associate the sequence $\left(T_{n}\right)$ of functions defined on $\mathbb{T}$ by $T_{n}(x)=T\left(q_{n} x\right)$. This appendix is devoted to the analysis of the partial sums

$$
m_{n}(x)=T_{1}(x)+\cdots+T_{n}(x), \quad n \geq 1 .
$$

The sequence $\left(m_{n}\right)$ defines a random walk in $\mathbb{Z}$ and we are particularly interested in the interaction between different walks. We say that $\left(m_{n}\right)$ has the level-crossing property if, for Lebesgue almost every $x, y \in \mathbb{T}$, there exist infinitely many $n$ such that $m_{n}(x)=m_{n}(y)$.

If we assume that $2 q_{n}$ divides $q_{n+1}$ then every plateau of $T_{n}$ contains exactly the same number of positive and negative plateaux of $T_{n+1}$. If this holds for every $n$ then, for any $s_{1}, \ldots, s_{n} \in\{-1,1\}$,

$$
\lambda\left(\left\{x \in \mathbb{T} ; T_{j}(x)=s_{j} \text { for } j=1, \ldots, n\right\}\right)=2^{-n}
$$

and so the $\left(T_{n}\right)$ are independent and identically distributed (i.i.d). In this case $\left(m_{n}\right)$ is a simple random walk in $\mathbb{Z}$, and thus the map that associates to each pair $(x, y) \in \mathbb{T} \times \mathbb{T}$ the process $\left(m_{n}(x)-m_{n}(y)\right)$ is a random walk in $\mathbb{Z}$ with finite support and zero mean. In particular, $\left(m_{n}(x)-m_{n}(y)\right)$ is recurrent almost surely (see $\S 4.2$ of [12]), and so $\left(m_{n}\right)$ has the level-crossing property.

The same might not be true if $2 q_{n}$ does not divide $q_{n+1}$. On the other hand, if $q_{n+1}$ is much larger than $q_{n}$, almost every plateau of $T_{n+1}$ is entirely contained inside a plateau of $T_{n}$ and so $\left(T_{n}\right)$ exhibits some sort of asymptotic independence. This is the content of the next result, which is used in Section 4 to prove ergodicity when one does not have the divisibility condition. The idea is to remove plateaux of $T_{n+1}$ not entirely contained inside plateaux of $T_{n}$ in such a way that independence holds in their complement.

Lemma A.1. Let $\left(q_{n}\right)$ be a sequence of positive integers and let $\left(T_{n}\right),\left(m_{n}\right)$ be as above. If

$$
\sum_{n \geq 1} \frac{q_{n}}{q_{n+1}}<\infty
$$

then $\left(m_{n}\right)$ has the level-crossing property.

Proof. We will construct a descending chain of Borel sets $\left(\Omega_{n}\right)$ of $\mathbb{T}$ such that, restricted to $\Omega_{n}$, the first $n$ functions $T_{1}, \ldots, T_{n}$ are i.i.d. A simple argument of induction will imply that the $\left(T_{n}\right)$ are i.i.d in the intersection $\Omega^{\infty}=\bigcap_{n \geq 1} \Omega_{n}$.

The construction is by induction. Let $\mathcal{F}_{n}$ be the family of plateaux of $T_{n}$ and $\mathcal{F}_{n}=\mathcal{F}_{n}^{+} \sqcup \mathcal{F}_{n}^{-}$its decomposition in positive and negative plateaux, respectively. Assume that $\Omega_{1}=\mathbb{T}, \ldots, \Omega_{n}$ have been constructed satisfying the following conditions.

(i) For $1 \leq j \leq n$, there is a set $\mathcal{G}_{j} \subset \mathcal{F}_{j}$ such that $\Omega_{j}=\bigcup_{J \in \mathcal{G}_{j}} J$. 
(ii) For $1 \leq i<j \leq n$, every element of $\mathcal{G}_{j}$ is contained in exactly one element of $\mathcal{G}_{i}$.

(iii) For any $s_{1}, \ldots, s_{n} \in\{-1,1\}$,

$$
\lambda\left(\left\{x \in \Omega_{n} ; T_{j}(x)=s_{j} \text { for } j=1, \ldots, n\right\}\right)=\frac{\lambda\left(\Omega_{n}\right)}{2^{n}} .
$$

Observe that (ii) automatically implies that $\left\{x \in \Omega_{n} ; T_{j}(x)=s_{j}\right.$ for $\left.j=1, \ldots, n\right\}$ is the union of elements of $\mathcal{G}_{n}$. Now let

$$
\mathcal{G}_{n+1}=\left\{J \in \mathcal{F}_{n+1} ; \exists I \in \mathcal{G}_{n} \text { such that } J \subset I\right\} \quad \text { and } \quad \Omega_{n+1}=\bigcup_{J \in \mathcal{G}_{n+1}} J .
$$

For each $I \in \mathcal{G}_{n}$, the number of elements of $\mathcal{G}_{n+1}$ entirely contained in $I$ is between $q_{n+1} / q_{n}-2$ and $q_{n+1} / q_{n}$. We may assume, removing at most two of these plateaux, that

$$
\#\left\{J \in \mathcal{G}_{n+1}^{+} ; J \subset I\right\}=\#\left\{J \in \mathcal{G}_{n+1}^{-} ; J \subset I\right\}
$$

and it is independent of $I$. (i) and (ii) are satisfied by definition. For (iii), fix $s_{1}, \ldots, s_{n} \in\{-1,1\}$ and let $\mathcal{G} \subset \mathcal{G}_{n}$ such that

$$
\left\{x \in \Omega_{n} ; T_{j}(x)=s_{j} \text { for } j=1, \ldots, n\right\}=\bigcup_{I \in \mathcal{G}} I .
$$

Then

$$
\left\{x \in \Omega_{n+1} ; T_{j}(x)=s_{j} \text { for } j=1, \ldots, n \text { and } T_{n+1}(x)=1\right\}=\bigcup_{\substack { I \in \mathcal{G} \\
\begin{subarray}{c}{J \in \mathcal{G}_{n+1}^{+} \\
J \subset I{ I \in \mathcal { G } \\
\begin{subarray} { c } { J \in \mathcal { G } _ { n + 1 } ^ { + } \\
J \subset I } }\end{subarray}} J
$$

has Lebesgue measure equal to

$$
\# \mathcal{G} \cdot \#\left\{J \in \mathcal{G}_{n+1}^{+} ; J \subset I\right\} \cdot \frac{1}{2 q_{n+1}},
$$

which is, by (A.1), independent of $s_{1}, \ldots, s_{n}$. Doing the same when $T_{n+1}(x)=-1$, (iii) is established.

The same argument applies to prove that, for $m \geq n$,

$$
\lambda\left(\left\{x \in \Omega_{m} ; T_{j}(x)=s_{j} \text { for } j=1, \ldots, n\right\}\right)=\frac{\lambda\left(\Omega_{m}\right)}{2^{n}}
$$

and so, letting $m \rightarrow \infty$,

$$
\lambda\left(\left\{x \in \Omega^{\infty} ; T_{j}(x)=s_{j} \text { for } j=1, \ldots, n\right\}\right)=\frac{\lambda\left(\Omega^{\infty}\right)}{2^{n}},
$$

proving that the $\left(T_{n}\right)$ are independent in $\Omega^{\infty}$.

Now we estimate $\lambda\left(\Omega^{\infty}\right)$. By construction, inside each $I \in \mathcal{G}_{n}$ at most 4 elements of $\mathcal{F}_{n+1}$ are removed and so

$$
\lambda\left(\bigcup_{\substack{J \in \mathcal{G}_{n+1} \\ J \subset I}} J\right) \geq \lambda(I)-4 \cdot \frac{1}{2 q_{n+1}} .
$$

Summing this up in $I$ yields

$$
\lambda\left(\Omega_{n+1}\right) \geq \lambda\left(\Omega_{n}\right)-\# \mathcal{G}_{n} \cdot \frac{2}{q_{n+1}} \geq \lambda\left(\Omega_{n}\right)-\frac{4 q_{n}}{q_{n+1}}
$$


and then

$$
\lambda\left(\Omega^{\infty}\right) \geq 1-4 \sum_{n \geq 1} \frac{q_{n}}{q_{n+1}} .
$$

If, instead of beginning the construction in step 1 we start in step $n_{0}$, the limit set $\Omega_{n_{0}}^{\infty}$ has Lebesgue measure at least $1-4 \sum_{n \geq n_{0}} q_{n} / q_{n+1}$. By construction, the map that sends $(x, y) \in \Omega_{n_{0}}^{\infty} \times \Omega_{n_{0}}^{\infty}$ to the process $\left(m_{n}(x)-m_{n}(y)\right)_{n \geq n_{0}}$ is a random walk in $\mathbb{Z}$ with finite support and zero mean, and thus it visits 0 infinitely often almost surely (see $\S 4.2$ of [12]).

Since $\bigcup_{n_{0} \geq 1} \Omega_{n_{0}}^{\infty}$ has full Lebesgue measure, it follows that $\left(m_{n}\right)$ has the levelcrossing property.

\section{Appendix B. A faCt on COntinued Fractions}

Remember the definition of Subsection 2.2, $\alpha \in \mathbb{R}$ is divisible if it has a sequence $\left(q_{n_{j}}\right)$ of continuants satisfying

$$
2 q_{n_{j}} \text { divides } q_{n_{j+1}} \text { and } \lim _{j \rightarrow \infty} q_{n_{j}}\left\|q_{n_{j}} \alpha\right\|=0 .
$$

In this appendix, we want to prove that

Proposition B.1. Lebesgue almost every $\alpha \in \mathbb{R}$ is divisible.

To prove it, we first collect an auxiliary lemma and identify a mechanism to guarantee the divisibility property. Once this is done, Proposition B.1 will follow. We acknowledge Carlos Gustavo Moreira for communicating us this proof.

For positive integers $a_{1}, \ldots, a_{n}$, we recall the continuant $K\left(a_{1}, \ldots, a_{n}\right)$ denotes the denominator of the rational number

$$
\left[0 ; a_{1}, \ldots, a_{n}\right]=\frac{1}{a_{1}+\frac{1}{a_{2}+\frac{1}{\ddots+\frac{1}{a_{n}}}}} .
$$

Lemma B.2. Let $n \geq 3, a_{1}, a_{2}, \ldots, a_{n-1}$ and $q$ be positive integers. Then there exist integers $a, b$ such that if

$$
\left\{\begin{array}{rlll}
a_{n} & \equiv a(\bmod q) \\
a_{n+1} & \equiv b \quad(\bmod q)
\end{array}\right.
$$

then $q$ divides $K\left(a_{1}, a_{2}, \ldots, a_{n}, a_{n+1}\right)$.

Proof. Let $a$ be the product of the primes that divide $q$ and do not divide neither of the continuants $K\left(a_{1}, a_{2}, \ldots, a_{n-2}\right), K\left(a_{1}, a_{2}, \ldots, a_{n-1}\right)$. If $a_{n} \equiv a(\bmod q)$, then

$$
K\left(a_{1}, a_{2}, \ldots, a_{n}\right)=a \cdot K\left(a_{1}, a_{2}, \ldots, a_{n-1}\right)+K\left(a_{1}, a_{2}, \ldots, a_{n-2}\right)
$$

and $q$ are coprime. This guarantees that, as $b$ varies modulo $q$, the number

$$
K\left(a_{1}, a_{2}, \ldots, a_{n}, a_{n+1}\right)=b \cdot K\left(a_{1}, a_{2}, \ldots, a_{n}\right)+K\left(a_{1}, a_{2}, \ldots, a_{n-1}\right)
$$

runs over all residues modulo $q$ and so, for one of these classes, it is divisible by $q$.

The auxiliary lemma concerns the following elementary facts about continued fractions and continuants.

Lemma B.3. Let $\alpha=\left[a_{0} ; a_{1}, a_{2}, \ldots\right]$ be an irrational number. 
(a) If $\left(q_{n}\right)$ is the sequence of continuants of $\alpha$, then

$$
\frac{1}{a_{n+1}+2}<q_{n}\left\|q_{n} \alpha\right\|<\frac{1}{a_{n+1}} .
$$

(b) The probability that $a_{n+1}=k$, given that $a_{1}=k_{1}, \ldots, a_{n}=k_{n}$, is between $\frac{1}{(k+1)(k+2)}$ and $\frac{2}{k(k+1)}$.

(c) The probability that $a_{n+1} \geq k$, given that $a_{1}=k_{1}, \ldots, a_{n}=k_{n}$, is between $\frac{1}{k+1}$ and $\frac{2}{k}$.

Proof. (a) is a well-known fact and can be checked in any introductory text of continued fractions. Let's prove (b). Once $a_{1}, \ldots, a_{n}$ are fixed, the number $\alpha=$ $\left[0 ; a_{1}, \ldots, a_{n}, \alpha_{n+1}\right]$ belongs to the interval with endpoints $\frac{p_{n}}{q_{n}}$ and $\frac{p_{n}+p_{n-1}}{q_{n}+q_{n-1}}$. In these conditions, $a_{n+1}=k$ if and only if $\alpha$ belongs to the interval of endpoints $\frac{k p_{n}+p_{n-1}}{k q_{n}+q_{n-1}}$ and $\frac{(k+1) p_{n}+p_{n-1}}{(k+1) q_{n}+q_{n-1}}$. Using the relation $\left|p_{n} q_{n-1}-p_{n-1} q_{n}\right|=1$, it follows that the ratio of the lengths of these two intervals is equal to

$$
\frac{q_{n}\left(q_{n}+q_{n-1}\right)}{\left[k q_{n}+q_{n-1}\right]\left[(k+1) q_{n}+q_{n-1}\right]}=\frac{1+\frac{q_{n-1}}{q_{n}}}{\left(k+\frac{q_{n-1}}{q_{n}}\right)\left(k+1+\frac{q_{n-1}}{q_{n}}\right)},
$$

which belongs to $\left[\frac{1}{(k+1)(k+2)}, \frac{2}{k(k+1)}\right]$. This establishes (b). To prove (c), just observe that

$$
\sum_{j \geq k} \frac{1}{(j+1)(j+2)}=\frac{1}{k+1} \text { and } \sum_{j \geq k} \frac{2}{j(j+1)}=\frac{2}{k} .
$$

Proof of Proposition B.1. For each positive integer $q$, let $D_{q}$ be the set of $\alpha \in \mathbb{R}$ for which there are infinitely many $n \in \mathbb{N}$ such that $q$ divides $q_{n}$ and $a_{n+1} \geq n$.

Claim. $D_{q}$ has full Lebesgue measure.

We prove this via the auxiliary lemmas. Assume that $a_{1}, a_{2}, \ldots, a_{3 k-1}$ are given. By Lemma B.2, there are $a, b \in\{1, \ldots, q\}$ such that $K\left(a_{1}, a_{2}, \ldots, a_{3 k-1}, a, b\right)$ is divisible by $q$. By Lemma B.3, the probability that $a_{3 k}=a, a_{3 k+1}=b$ and $a_{3 k+2} \geq 3 k+1$ is at least $\frac{1}{(q+1)^{2}(q+2)^{2}(3 k+1)}$. Thus, given $k_{0} \geq 1$, the probability that, for each $k \geq k_{0}$, either $q_{n}$ is not a multiple of $q$ or $a_{n+1}<n$, is at most

$$
\prod_{k \geq k_{0}}\left(1-\frac{1}{(q+1)^{2}(q+2)^{2}(3 k+1)}\right)=0 .
$$

This proves the claim.

To conclude the proof of the proposition, consider the intersection $\bigcap_{q \geq 1} D_{q}$, which by the above claim has full Lebesgue measure. Each $\alpha \in \bigcap_{q \geq 1} D_{q}$ is divisible. Indeed, one can inductively construct a sequence $\left(n_{j}\right)$ such that $2 q_{n_{j}}$ divides $q_{n_{j+1}}$ and $a_{n_{j}+1} \geq n_{j}$. Observing that, by Lemma B.3.

$$
\lim _{j \rightarrow \infty} q_{n_{j}}\left\|q_{n_{j}} \alpha\right\| \leq \lim _{j \rightarrow \infty} \frac{1}{a_{n_{j}+1}}=0,
$$

the proof is complete. 
Remark B.4. The above argument, together with the fact that, for Lebesgue almost every $\alpha \in \mathbb{R},\left(q_{n}\right)$ grows at most (and at least) exponentially fast, can be used to show that Lebesgue almost every $\alpha \in \mathbb{R}$ has a sequence of continuants $\left(q_{n_{j}}\right)$ such that $2 q_{n_{j}}$ divides $q_{n_{j+1}}$ and

$$
q_{n_{j}}\left\|q_{n_{j}}\right\|<\frac{1}{\log q_{n_{j}}}
$$

\section{REFERENCES}

[1] J. Aaronson, Rational ergodicity and a metric invariant for Markov shifts, Israel J. Math. 27 (1977), no. 2, 93-123.

[2] J. Aaronson, Ergodic theory for inner functions of the upper half plane, Ann. Inst. H. Poincar Sect. B (N.S.) 14 (1978), no. 3, 233-253.

[3] J. Aaronson, An introduction to infinite ergodic theory, Mathematical surveys and monographs 50 (1997), American Mathematical Society, Providence, RI.

[4] J. Aaronson, M. Denker and A. Fisher, Second order ergodic theorems for ergodic transformations of infinite measure spaces, Proc. Amer. Math. Soc. 114 (1992), no. 1, 115127.

[5] J. Aaronson and M. Keane, The visits to zero of some deterministic random walks, Proc. London Math. Soc. 44 (1982), no. 3, 535-553.

[6] J. Aaronson, M. Lemanćzyk, C. Mauduit, and H. Nakada, Koksma's inequality and group extensions of Kronecker transformations, Algorithms, fractals, and dynamics (Okayama/Kyoto, 1992), 27-50, Plenum, New York (1995).

[7] J. Aaronson and D. Sullivan, Rational ergodicity of geodesic flows, Ergodic Theory \& Dynamical Systems 4 (1984), no. 2, 165-178.

[8] D. Anosov and A. Katok, New examples in smooth ergodic theory. Ergodic diffeomorphisms, Transactions of the Moscow Mathematical Society 23 (1970), 1-35.

[9] L. Baggett and K. Merrill, Smooth cocycles for an irrational rotation, Israel J. Math. 79 (1992), no. 2-3, 281-288.

[10] J.-P. Conze, Ergodicité d'une transformation cylindrique (French), Bull. Soc. Math. France 108 (1980), no. 4, 441-456.

[11] J.-P. Conze and M. Keane, Ergodicité d'un flot cylindrique (French), Séminaire de Probabilités I, Exp. No. 5, 7 pp. Dépt. Math. Informat., Univ. Rennes, Rennes (1976).

[12] R. Durrett, Probability: theory and examples, The Wadsworth \& Brooks/Cole Statistics/Probability Series, Pacific Grove (1991).

[13] B. Fayad and M. Lemańczyk, On the ergodicity of cylindrical transformations given by the logarithm, Mosc. Math. J.6 (2006), no. 4, 657-672, 771-772.

[14] K. Frączek, On ergodicity of some cylinder flows, Fund. Math. 163 (2000), no. 2, 117-130.

[15] A. Katok, Combinatorial constructions in ergodic theory and dynamics, University Lecture Series 30. American Mathematical Society, Providence ,RI (2003).

[16] A. Krygin, Examples of ergodic cylindrical cascades (Russian), Mat. Zametki 16 (1974), 981-991.

[17] F. Ledrappier and O. Sarig, Unique ergodicity for non-uniquely ergodic horocycle flows, Discrete Contin. Dyn. Syst. 16 (2006), no. 2, 411-433.

[18] I. Oren, Ergodicity of cylinder flows arising from irregularities of distribution, Israel J. Math. 44 (1983), no. 2, 127-138.

[19] D. Pask, Ergodicity of certain cylinder flows, Israel J. Math. 76 (1991), no. 1-2, 129-152.

[20] O. Sarig, Unique ergodicity for infinite measures, Proc. Inter. Congress Math., Hyderabad (2010).

[21] K. Schmidt, Cocycles on ergodic transformation groups, Macmillan Lectures in Mathematics, vol. 1, Macmillan Company of India (1977).

[22] K. Schmidt, A cylinder flow arising from irregularity of distribution, Compositio Math. 36 (1978), no. 3, 225-232. 
Universidade Estadual Paulista, Rua Cristóvão Colombo 2265, 15054-000, São José do Rio Preto, Brasil.

E-mail address: prcirilo@ibilce.unesp.br

Weizmann Institute of Science, Faculty of Mathematics and Computer Science, POB 26, 76100, Rehovot, IsRAel.

E-mail address: yuri.lima@weizmann.ac.il

Instituto Nacional de Matemática Pura e Aplicada, Estrada Dona Castorina 110, 22460-320, Rio DE JANeiro, Brasil.

E-mail address: enrique@impa.br 